\title{
Strong localized asperity of the 2011 Tohoku-Oki earthquake, Japan
}

\author{
Hiroyuki Kumagai, Nelson Pulido, Eiichi Fukuyama, and Shin Aoi \\ National Research Institute for Earth Science and Disaster Prevention, Tsukuba, Ibaraki 305-0006, Japan
}

(Received October 7, 2011; Revised January 19, 2012; Accepted January 21, 2012; Online published August 16, 2012)

\begin{abstract}
Our moment tensor inversion of the 2011 Tohoku-Oki earthquake, using regional broadband strong-motion waveforms, indicates that the earthquake can be approximated by a point source. The amplitude spectra of the observed displacement seismograms were fitted by the omega-square model, which resulted in the corner frequency of around $0.016 \mathrm{~Hz}$. This implies a large slip over a circular fault having a radius of $70 \mathrm{~km}$, with a rupture duration of about $40 \mathrm{~s}$. The moment-rate function estimated from the inversion shows a large impulse of similar duration. We interpret this impulse to correspond to the rupture estimated from the corner frequency. From the seismic moment released during the impulse, we have estimated the average slip and stress drop over the fault to be $50 \mathrm{~m}$ and $40 \mathrm{MPa}$, respectively. This stress drop corresponds to an effective normal stress larger than $200 \mathrm{MPa}$, indicating that a strong localized asperity (mega asperity) was ruptured during the earthquake. Previous simulation studies suggested the importance of a large effective normal stress at a shallow plate interface, which was explained by a pore pressure distribution along the plate. We have explored the possibility of a subducted seamount to be the origin of the mega asperity.
\end{abstract}

Key words: Waveform inversion, centroid moment tensor, stress drop, effective normal stress, seamount.

\section{Introduction}

One of the serious questions raised by the Tohoku-Oki earthquake of 11 March, 2011, is why the mega earthquake occurred in the subduction zone along the Japan Trench, where the plates were believed to be weakly coupled (e.g., Kanamori, 1986). Various slip models for the Tohoku-Oki earthquake have been proposed from seismic, GPS, and tsunami, data analyses (e.g., Fujii et al., 2011; Ide et al., 2011; Lay et al., 2011; Ozawa et al., 2011; Simons et al., 2011). However, these slip models display a large variability, which may reflect the non-uniqueness of an underdetermined inverse problem of solving the slip distribution over a finite fault (e.g., Simons et al., 2011). On the other hand, recent models using ocean-bottom GPS and deformation data (Ito et al., 2011; Sato et al., 2011) point to a large coseismic slip of more than $60 \mathrm{~m}$ near the hypocenter. The occurrence of such a large slip implies a large stress drop associated with the earthquake.

To characterize the earthquake source, we have adopted here a simple approach of waveform inversion based on a point source moment tensor representation using regional broadband strong-motion waveforms. This approach avoids the under-determined problem, and may be used to identify the large stress drop rupture area in a way that is different from previous studies. Our moment-tensor inversion and spectral analysis, based on the omega-square model, point to a localized asperity over a circular fault having a radius of $70 \mathrm{~km}$ with an average slip and stress drop of $50 \mathrm{~m}$ and

Copyright (C) The Society of Geomagnetism and Earth, Planetary and Space Sciences (SGEPSS); The Seismological Society of Japan; The Volcanological Society of Japan; The Geodetic Society of Japan; The Japanese Society for Planetary Sciences; TERRAPUB.

doi:10.5047/eps.2012.01.004
$40 \mathrm{MPa}$, respectively. The stress drop corresponds to an effective normal stress larger than $200 \mathrm{MPa}$. Such a large effective normal stress is consistent with that inferred from the numerical simulation study of Kato and Yoshida (2011), who suggested the significance of a shallow strong asperity on the occurrence of the Tohoku-Oki earthquake. Yoshida and Kato (2011) explained the large effective normal stress by a pore pressure distribution along the plate. The strong localized asperity may be alternatively explained by a subducted seamount, which increases plate coupling locally (Scholz and Small, 1997). In this paper, we present the results of our analysis of the Tohoku-Oki earthquake, and discuss the origin of the shallow strong asperity.

\section{Data and Inversion Method}

We used broadband strong-motion seismograms from the nationwide broadband seismic network of the F-net, installed and operated by the National Research Institute for Earth Science and Disaster Prevention (NIED) (e.g., Fukuyama et al., 2001). At each F-net station, a velocitytype strong-motion seismometer is installed at the end of a 30-50-m-long vault to record ground velocities up to $\pm 2 \mathrm{~m} / \mathrm{s}$.

We used the waveform inversion method developed by Nakano et al. (2008), in which the inverse problem was solved in the frequency domain. A double-couple focal mechanism was assumed for a point source in the inversion. A grid search in space was conducted to find the combination of the best-fit fault and slip orientation parameters (strike, dip, and rake angles) and source location. We obtained the moment function in the time domain from the inverse Fourier transform of the frequency components determined by the inversion. The moment function obtained in this way was a band-passed form since we used band-passed 

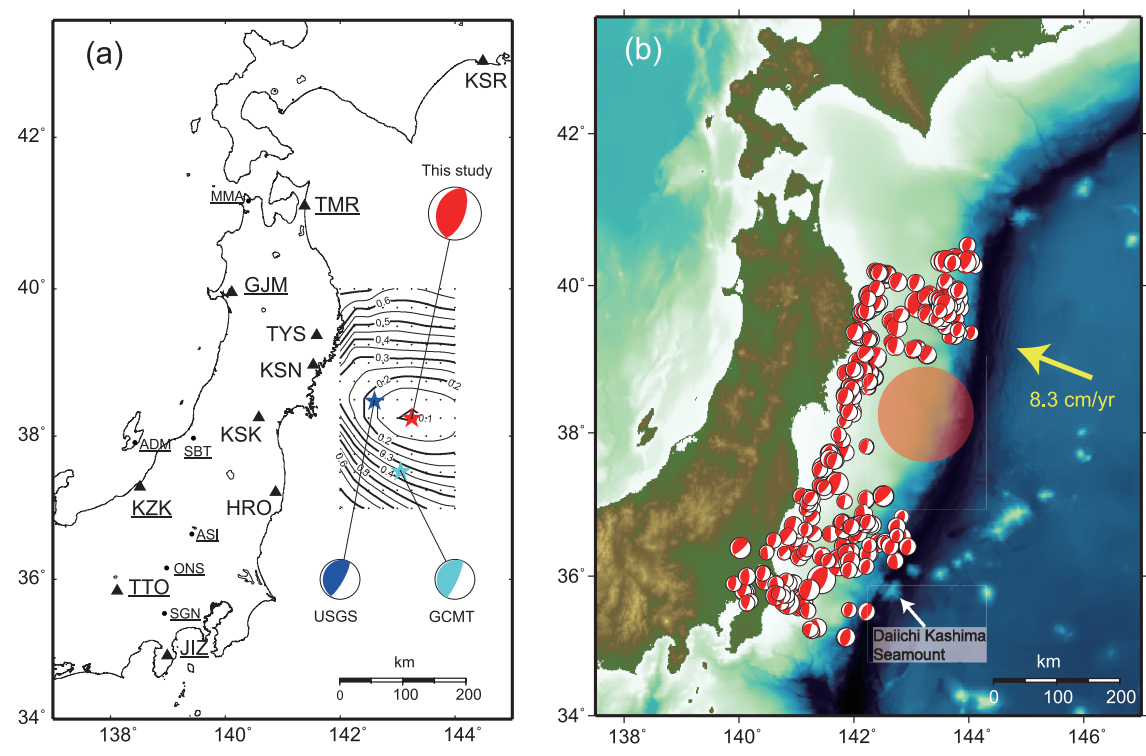

Fig. 1. (a) Map around the source area of the Tohoku-Oki earthquake. The focal mechanism at the red star indicates the best-fit solution obtained from our waveform inversion of the Tohoku-Oki earthquake. Solid black triangles show the locations of stations used in the inversion. Small black dots denote node points for the spatial grid search, and contour lines represent the residuals at a depth of $30 \mathrm{~km}$. The focal mechanisms at the blue and cyan stars are those determined by the Global CMT project and U.S. Geological Survey. Station codes with underlines are the stations used in the corner frequency analysis. (b) Map showing bathymetry along the Japan Trench and topography of northeast Japan. The red circle represents the source area of the Tohoku-Oki earthquake estimated by this study. The yellow arrow indicates the convergence rate vector of the Pacific Plate with respect to the North American Plate. Focal mechanisms represent aftershocks with thrust type mechanisms that occurred within the subducted plate (Asano et al., 2011).

observed waveforms for the inversion. A deconvolved form of the moment function was estimated by fitting a function represented by a superposition of elementary functions to a band-passed form of the moment function determined by the inversion.

We used broadband strong-motion records with a sampling frequency of $20 \mathrm{~Hz}$ at $10 \mathrm{~F}$-net stations (Fig. 1(a)). The selection of these stations was based on signal-to-noise ratios at low frequencies and to have a wide azimuthal coverage to the source. After instrumental corrections, we applied a band-pass filter between 0.006 and $0.02 \mathrm{~Hz}$ and adopted a sampling frequency of $1 \mathrm{~Hz}$. We used a total data length of $512 \mathrm{~s}$ starting from 14:46:18 on 11 March, 2011, (Japan standard time) for the inversion. Grid nodes were positioned at horizontal (EW and NS) and vertical intervals of $0.25^{\circ}$ and $10 \mathrm{~km}$, respectively (Fig. 1(a)). Green's functions were calculated with the structure used by routine centroid moment tensor determinations by NIED (Kubo et al., 2002). In the reconstruction of a deconvolved form of the moment function, we used a step-like function as the elementary function, and their time interval, and initial and final times, were determined by the Akaike Information Criterion (see Nakano et al. (2008) for details).

\section{Results}

Our waveform inversion indicates that the observed strong-motion waveforms at regional distances are well explained by a point source modeling (Fig. 2). The bestfit source location was estimated at $38.25^{\circ} \mathrm{N}, 143.25^{\circ} \mathrm{E}$, at a depth of $30 \mathrm{~km}$, at which the normalized residual was 0.093 (variance reduction of $90.7 \%$ ). The best-fit focal mechanism was represented by the fault parameters (strike, dip, rake) $=(200,30,90)$ (Fig. 1(a)). The esti- mated mechanism is consistent with those estimated from global broadband seismographic network data by the global CMT project and the National Earthquake Information Center (NEIC) of the U.S. Geological Survey, although the centroid source location and dip angle are slightly different from the global data estimations (Fig. 1(a)). The estimated seismic moment was $4.8 \times 10^{22} \mathrm{~N} \mathrm{~m}\left(M_{\mathrm{w}}=9.1\right)$ (Fig. 3(a)). The moment-rate function (Fig. 3(b)) shows a source duration of roughly $200 \mathrm{~s}$ and a peak moment rate at 50-100 s from the source origin time. Our inversion results indicate that the point source assumption is satisfied in view of the observations at regional distances.

To estimate the source size and the amount of slip, we used the spectra of the observed displacement seismograms at low frequencies, which can be represented by the omega-square model in the far field (Aki, 1967). We estimated the corner frequency of the omega-square model in the following way. A displacement seismogram was corrected for medium attenuation using the following equation: $u_{\mathrm{c}}(\omega)=u(\omega) e^{B l}$, where $u$ and $u_{\mathrm{c}}$ are the observed and corrected displacements, respectively; $\omega$ is angular frequency; and $l$ is the hypocentral distance. Here, $B$ is given by $B=\omega /\left(2 Q \beta_{0}\right)$, where $Q$ is the quality factor for medium attenuation and $\beta_{0}$ is an $S$-wave velocity at shallow depths. We used $\beta_{0}=3500 \mathrm{~m} / \mathrm{s}$ and $Q=300$. The amplitude spectrum of a corrected displacement seismogram was fitted by the following omega-square model (Aki, 1967): $S(\omega)=S(0) /\left[1+\left(\omega / \omega_{\mathrm{c}}\right)^{2}\right]$, where $\omega_{\mathrm{c}}=2 \pi f_{\mathrm{c}}$ and $f_{\mathrm{c}}$ is the corner frequency.

Assuming slip on a circular crack and a rupture velocity of $0.8 \beta$, where $\beta$ is an $S$-wave velocity at the source, the corner frequency $f_{\mathrm{c}}$, for $S$ waves of the omega-square model, is related to the crack radius $r$ by the equation 


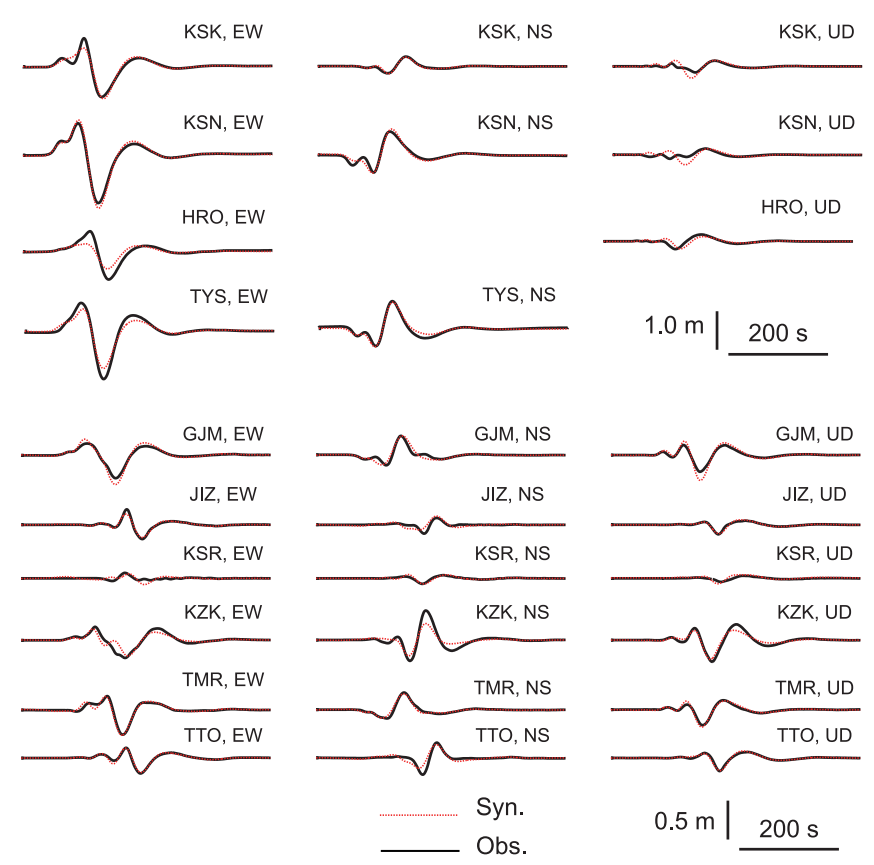

Fig. 2. Waveform fits obtained from the waveform inversion of the Tohoku-Oki earthquake. Black solid and red dotted lines represent the observed and synthetic waveforms, respectively. Waveforms with low signal-to-noise ratios, which were not used in the inversion, are not shown.
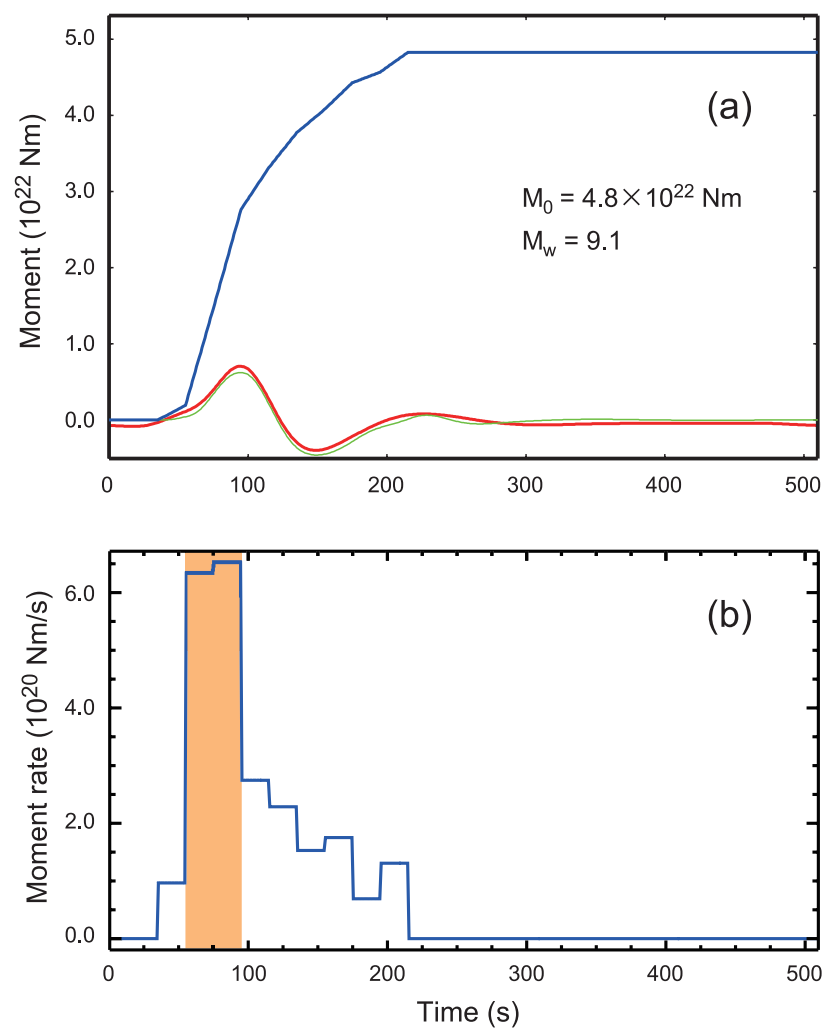

Fig. 3. (a) Deconvolved form of the moment function (blue line) estimated by fitting the function represented by a superposition of elementary functions (green line) to the band-passed form of the moment function determined by the inversion (red line). (b) Moment-rate function estimated from the moment function shown in Fig. 3(a). The orange bar represents the duration of the peak pulse in the moment-rate function (see text for details).

$f_{\mathrm{c}}=0.3 \beta / r$ (Sato and Hirasawa, 1973). To estimate the corner frequency, we selected stations located at an intermediate distance from the source. This is because seismograms at closer stations may be contaminated by $P$ waves and near-field effects and those at distant stations are affected by surface waves. Of the 10 stations used in the waveform inversion, we selected 5 stations (TMR, GJM, KZK, TTO, and JIZ). Since the estimation of the corner frequency is strongly affected by noise, we used additional stations (MMA, SBT, ADM, ASI, ONS, and SGN) (Fig. 1(a)) to obtain a stable estimation. Figures 4(a) and 4(b) show the original and band-passed $(0.006-0.02 \mathrm{~Hz})$ vertical velocity 

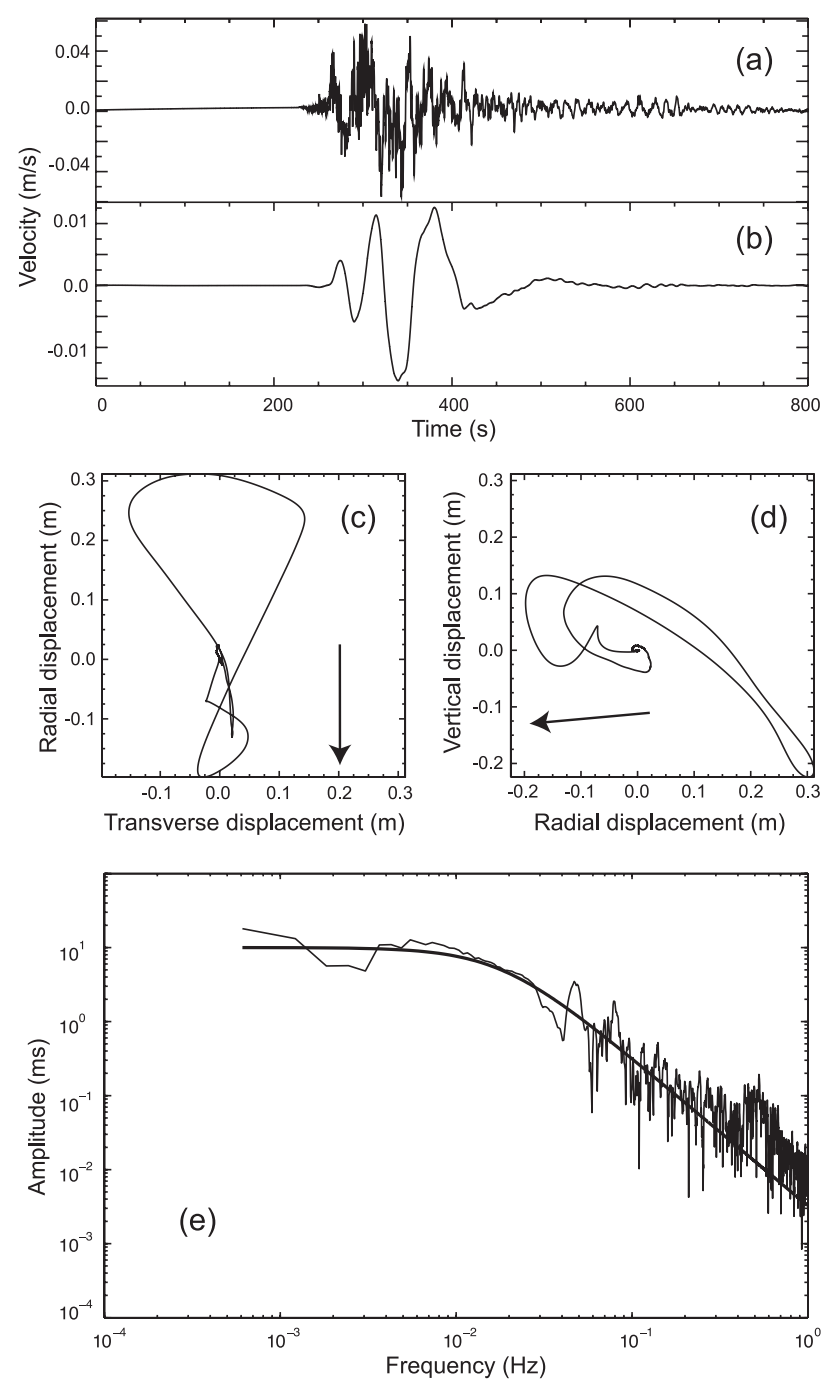

Fig. 4. (a) Original and (b) band-passed $(0.006-0.02 \mathrm{~Hz})$ vertical ground velocity seismograms at GJM. (c, d) Particle motions of band-passed $(0.006-0.02 \mathrm{~Hz})$ displacement seismograms at GJM in the radial-transverse and vertical-radial planes, respectively. Arrows in Figs. 4(c) and 4(d) indicate the direction to the earthquake source. (e) Amplitude spectrum of the vertical displacement seismogram corrected for medium attenuation (thin solid line) and the best fit of the omega-square model to the spectrum (thick solid line) at GJM.

seismograms at GJM, respectively. In view of the bandpassed displacement particle motions in Figs. 4(c) and 4(d), the dominant wave recorded in these seismograms is an $S V$ wave, which justifies the use of the omega-square model for $S$ waves. As shown in Fig. 4(e), the omega-square model reasonably represents the spectrum of the observed data. We estimated $f_{\mathrm{c}}$ values using the vertical and radial seismograms at 11 stations (Fig. 1(a)). The average value of $f_{\mathrm{c}}$ was $0.016 \pm 0.005 \mathrm{~Hz}$. Since the signal-to-noise ratios of aftershocks at low frequencies were poor, the spectral ratio technique could not be applied in this case.

Using $f_{\mathrm{c}}=0.016 \mathrm{~Hz}$ and $\beta=3800 \mathrm{~m} / \mathrm{s}$ at the source, we obtain $r \cong 70 \mathrm{~km}$. The rupture duration $\tau$ for a circular crack rupture including the stopping phase may be given by $\tau=2 r /(0.8 \beta)$. Using this equation, we obtain $\tau \cong 40 \mathrm{~s}$ for $r=70 \mathrm{~km}$. This duration coincides with the peak pulse duration in the moment-rate function in Fig. 3(b) (orange bar). We therefore conclude that the peak pulse corresponds to the rupture estimated by the corner frequency. The seismic moment for the peak pulse in the moment-rate function is $2.8 \times 10^{22} \mathrm{~N} \mathrm{~m}$, which is $60 \%$ of the total seismic moment.
The remaining $40 \%$ of the seismic moment may correspond to rupture beyond the crack.

The seismic moment $M_{0}$ for a circular crack is defined by the equation $M_{0}=\mu A D$, where $\mu$ is the rigidity, $A=\pi r^{2}$ is the slip area, and $D$ is the average slip. Using $r=70 \mathrm{~km}$, $\mu=40 \mathrm{GPa}$, and $M_{0}=2.8 \times 10^{22} \mathrm{~N} \mathrm{~m}$ for the peak pulse, we obtain $D \cong 50 \mathrm{~m}$. The stress drop $\Delta \sigma$ within the crack is estimated to be about $40 \mathrm{MPa}$ following the equation $\Delta \sigma=(7 / 16) M_{0} / r^{3}$ (Sato and Hirasawa, 1973). This value is anomalously large compared to those of ordinary interplate earthquakes, which are in a range of 1-10 $\mathrm{MPa}$ (e.g., Kanamori and Anderson, 1975). The stress drop is given as $\Delta \sigma=\sigma_{\mathrm{i}}-\sigma_{\mathrm{f}}$, where $\sigma_{\mathrm{i}}$ and $\sigma_{\mathrm{f}}$ are the initial and final shear stresses on the fault, respectively. We define the yield stress $\sigma_{\mathrm{s}}=\mu_{\mathrm{s}} \sigma_{\mathrm{n}}^{\mathrm{e}}$, where $\mu_{\mathrm{s}}$ is the static friction coefficient and $\sigma_{\mathrm{n}}^{\mathrm{e}}$ is the effective normal stress, and the dynamic stress $\sigma_{\mathrm{d}}=\mu_{\mathrm{d}} \sigma_{\mathrm{n}}^{\mathrm{e}}$, where $\mu_{\mathrm{d}}$ is the dynamic friction coefficient. If we assume that $\sigma_{\mathrm{f}} \cong \sigma_{\mathrm{d}}, \sigma_{\mathrm{s}}-\sigma_{\mathrm{i}}=\sigma_{\mathrm{i}}-\sigma_{\mathrm{d}}$, and $\mu_{\mathrm{d}}=0.5 \mu_{\mathrm{s}}$ (Aochi and Kato, 2010), we obtain the relation $\Delta \sigma=0.25 \mu_{\mathrm{s}} \sigma_{\mathrm{n}}^{\mathrm{e}}$. If one assumes $\mu_{\mathrm{s}}=0.7$ as inferred from laboratory experiments (e.g., Byerlee, 1978), $\Delta \sigma=$ 
$40 \mathrm{MPa}$ yields $\sigma_{\mathrm{n}}^{\mathrm{e}}=230 \mathrm{MPa}$, which is consistent with the effective normal stress inferred by Kato and Yoshida (2011).

\section{Discussion}

Asano et al. (2011) has indicated that there exists a region where no aftershocks with thrust focal mechanisms occurred near the source area of the Tohoku-Oki earthquake, and concluded that this region corresponds to the large coseismic slip area. As shown in Fig. 1(b), our estimated asperity size is consistent with this region, which supports our estimation of the rupture size. The Geospatial Information Authority of Japan (GSI) performed a joint slip inversion of co-seismic GPS data from the Japanese nationwide GPS network (GEONET) and at ocean-bottom GPS stations near the epicentral region (Sato et al., 2011). The joint inversion result indicated a localized area $(L \cong 150 \mathrm{~km})$ having a slip larger than $40 \mathrm{~m}$, and a maximum slip of about $60 \mathrm{~m}$ near the hypocenter (see http://www.gsi.go.jp/cais/topic110520index.html). Ito et al. (2011) estimated a coseismic slip of roughly $80 \mathrm{~m}$ based on ocean-bottom deformation measurements above the source region. Therefore, our seismic estimations of the large slip area $(L=2 r \cong 140 \mathrm{~km})$ and the average $\operatorname{slip}(D \cong 50 \mathrm{~m})$ are consistent with these other studies.

Kanamori and Astiz (1985) showed that the ratio $\eta$ of seismic slip to the total plate motion varies systematically as a function of the age of the subducting plate. Values of $\eta$ for subduction zones with relatively young plates are close to 1 , but $\eta$ is very small for the northeastern Japan subduction zone, indicating that the plates are almost decoupled. The occurrence of the mega earthquake is apparently not compatible with weak seismic coupling. Our results, indicating a large effective normal stress, imply a strong coupling in the mega asperity.

The estimated large effective normal stress (>200 MPa) is consistent with the numerical simulation study of Kato and Yoshida (2011). They indicated that a strong asperity at a shallow plate interface can reproduce the occurrence of great earthquakes similar to the Tohoku-Oki earthquake. Yoshida and Kato (2011) explained the shallow strong asperity with a pore pressure distribution along the subducted plate. They claimed that the large effective normal stress is achieved by a nearly hydrostatic pore pressure at a shallow part of the plate.

The shallow strong asperity may be alternatively explained by a subducted seamount. Scholz and Small (1997) proposed that subduction of a large seamount increases the normal stress across the subduction interface, which produces local seismic coupling in decoupled plates. The estimated effective normal stress ( $>200 \mathrm{MPa}$ ) may be achieved by a subducted seamount with a base width of $60 \mathrm{~km}$ and height of $4 \mathrm{~km}$ (typical dimensions of large seamounts) as shown by Schloz and Small (1997).

The two-dimensional model of Kato and Yoshida (2011) does not explain the lateral localization of the asperity. On the other hand, the seamount hypothesis naturally explains the localized asperity and the occurrence of the mega earthquake in the decoupled plates. The tomographic model of Zhao et al. (2011) displays velocity anomalies in the source region of the Tohoku-Oki earthquake. Similar velocity anomalies are also seen in the tomographic image of Matsubara and Obara (2011). Both Zhao et al. (2011) and Matsubara and Obara (2011) suggested a subducted seamount to be the origin of the velocity anomalies.

On the other hand, no clear structural features indicating a subducted seamount are recognizable in seismic reflection sections along the Japan Trench (e.g., Tsuru et al., 2002). Furthermore, Mochizuki et al. (2008) concluded that interplate coupling is weak over a large seamount known as the Daiichi-Kashima Seamount, that is being subducted at the trench off Ibaraki (Fig. 1(b)). Wang and Bilek (2011) suggested that extensive fracturing and faulting in the upper plate accompanied by the uplift due to a subducted seamount provide unfavorable conditions for the generation and propagation of large ruptures. However, the fracturing and faulting may depend on the rheological property of the plate, which was not considered in their study. Further structural investigations and theoretical studies are required to verify the validity of the seamount hypothesis.

In summary, our waveform inversion and spectral analysis, based on the omega-square model, show that the shallow strong localized asperity was ruptured during the Tohoku-Oki earthquake. The pore pressure hypothesis has a difficulty in explaining the localized nature of the asperity, and there is very little supporting evidence for the seamount hypothesis. Future studies are necessary to identify the origin of the shallow strong localized asperity, which is a key to understanding the source process of the Tohoku-Oki earthquake.

Acknowledgments. Comments from Nathan Bangs, an anonymous reviewer, and Kiyoshi Yomogida helped to improve the manuscript.

\section{References}

Aki, K., Scaling law of seismic spectrum, J. Geophys. Res., 72, 1217-1231, 1967.

Aochi, H. and A. Kato, Dynamic rupture of crosscutting faults: A possible rupture process for the $2007 M_{w} 6.6$ Niigata-ken Chuetsu-Oki earthquake, J. Geophys. Res., 115, B05310, doi:10.1029/2009JB006556, 2010.

Asano, Y., T. Saito, Y. Ito, K. Shiomi, H. Hirose, T. Matsumoto, S. Aoi, S. Hori, and S. Sekiguchi, Spatial distribution and focal mechanisms of aftershocks of the 2011 off the Pacific coast of Tohoku Earthquake, Earth Planets Space, 63, 669-673, 2011.

Byerlee, J. D., Friction of rocks, Pure Appl. Geophys., 116, 615-626, 1978. Fujii, Y., K. Satake, S. Sakai, M. Shinohara, and T. Kanazawa, Tsunami source of the 2011 off the Pacific coast of Tohoku Earthquake, Earth Planets Space, 63, 815-820, 2011.

Fukuyama, E., A. Kubo, H. Kawai, and K. Nonomura, Seismic remote monitoring of stress field, Earth Planets Space, 53, 1021-1026, 2001.

Ide, S., A. Baltay, and G. C. Beroza, Shallow dynamic overshoot and energetic deep rupture in the $2011 \mathrm{Mw} 9.0$ Tohoku-Oki Earthquake, Science, 332, 1426-1429, doi:10.1126/science.1207020c, 2011.

Ito, Y., T. Tsuji, Y. Osada, M. Kido, D. Inazu, Y. Hayashi, H. Tsushima, R. Hino, and H. Fujimoto, Frontal wedge deformation near the source region of the 2011 Tohoku-Oki earthquake, Geophys. Res. Lett., 38, L00G05, doi:10.1029/2011GL048355, 2011.

Kanamori, H., Rupture process of subduction-zone earthquakes, Ann. Rev. Earth Planet. Sci., 14, 293-322, 1986.

Kanamori, H. and D. L. Anderson, Theoretical basis of some empirical relations in seismology, Bull. Seismol. Soc. Am., 65, 1073-1095, 1975.

Kanamori, H. and L. Astiz, The 1983 Akita-Oki earthquake and its implications for systematic of subduction earthquakes, Earthq. Predict. Res., 3, 305-317, 1985.

Kato, N. and S. Yoshida, A shallow strong patch model for the 2011 great 
Tohoku-oki earthquake: A numerical simulation, Geophys. Res. Lett., 38, L00G04, doi:10.1029/2011GL048565, 2011.

Kubo, A., E. Fukuyama, H. Kawai, and K. Nonomura, NIED seismic moment tensor catalogue for regional earthquakes around Japan: Quality test and application, Tectonophysics, 356, 23-48, 2002.

Lay, T., Y. Yamazaki, C. J. Ammon, K. F. Cheung, and H. Kanamori, The $2011 M_{\mathrm{w}} 9.0$ off the Pacific coast of Tohoku Earthquake: Comparison of deep-water tsunami signals with finite-fault rupture model predictions, Earth Planets Space, 63, 797-801, 2011.

Matsubara, M. and K. Obara, The 2011 off the Pacific coast of Tohoku Earthquake related to a strong velocity gradient with the Pacific plate, Earth Planets Space, 63, 663-667, 2011.

Mochizuki, K., T. Yamada, M. Shinohara, Y. Yamanaka, and T. Kanazawa, Weak interplate coupling by seamounts and repeating M 7 earthquakes, Science, 321, 1194-1197, doi:10.1126/science.1160250, 2008.

Nakano, M., H. Kumagai, and H. Inoue, Waveform inversion in the frequency domain for the simultaneous determination of earthquake source mechanism and moment function, Geophys. J. Int., 173, 1000-1011, doi:10.1111/j.1365-246X.2008.03783.x, 2008.

Ozawa, S., T. Nishimura, H. Suito, T. Kobayashi, M. Tobita, and T. Imakiire, Coseismic and postseismic slip of the 2011 magnitude- 9 TohokuOki earthquake, Nature, 475, 373-376, doi:10.1038/nature10227, 2011.

Sato, M., T. Ishikawa, N. Ujihara, S. Yoshida, M. Fujita, M. Mochizuki, and A. Asada, Displacement Above the hypocenter of the 2011 TohokuOki earthquake, Science, 332, 1395, doi:10.1126/science.1207401, 2011.
Sato, T. and T. Hirasawa, Body wave spectra from propagating shear cracks, J. Phys. Earth, 21, 415-431, 1973.

Scholz, C. H. and C. Small, The effect of seamount subduction on seismic coupling, Geology, 25, 487-490, 1997.

Simons, M. et al., The 2011 magnitude 9.0 Tohoku-Oki Earthquake: Mosaicking the megathrust from seconds to centuries, Science, 332, 14211425, doi:10.1126/science.1206731, 2011.

Tsuru, T., J.-O. Park, S. Miura, S. Kodaira, Y. Kido, and T. Hayashi, Alongarc structural variation of the plate boundary at the Japan Trench margin: Implication of interplate coupling, J. Geophys. Res., 107(B12), 2357, doi:10.1029/2001JB001664, 2002.

Wang, K. and S. L. Bilek, Do subducting seamounts generate or stop large earthquakes?, Geology, 39, 819-822, doi:10.1130/G31856.1, 2011.

Yoshida, S. and N. Kato, Pore pressure distribution along plate interface that causes a shallow asperity of the 2011 great Tohoku-oki earthquake, Geophys. Res. Lett., 38, L00G13, doi:10.1029/2011GL048902, 2011.

Zhao, D., Z. Huang, N. Umino, A. Hasegawa, and H. Kanamori, Structural heterogeneity in the megathrust zone and mechanism of the 2011 Tohoku-oki earthquake $\left(M_{w}\right.$ 9.0), Geophys. Res. Lett., 38, L17308, doi:10.1029/2011GL048408, 2011.

H. Kumagai (e-mail: kumagai@bosai.go.jp), N. Pulido, E. Fukuyama, and S. Aoi 